\title{
Rake-Based Multiuser Detection for Quasi-Synchronous SDMA Systems
}

\author{
Shaodan Ma, Yonghong Zeng, and Tung-Sang Ng, Fellow, IEEE
}

\begin{abstract}
In this letter, a Rake-based multiuser detection technique, consisting of multiuser single-path signal separation, time-delay estimation, and multipath combining, is proposed for quasi-synchronous spatial-division multiple-access (SDMA) systems. Time diversity is achieved for performance improvement. In addition, only the upper bounds of the channel length and the time delays are required. Simulation results verify the effectiveness of the proposed technique, as well as its robustness against overestimation of the maximum channel length and the maximum time delay.
\end{abstract}

Index Terms-Multiuser detection, Rake receiver, spatialdivision multiple access (SDMA).

\section{INTRODUCTION}

W ITH SYSTEM capacity increasing linearly with the number of antennas, spatial-division multiple access (SDMA) is an important technique for future multiuser wireless communications [1]-[6]. One potential application of SDMA is in the uplink of a cellular mobile communication system. For this application, perfect synchronization among all users is generally difficult to achieve, due to different locations of mobiles and multipath propagations. A quasi-synchronous SDMA system operating in multipath channels is therefore considered here. This is a practical situation for cellular systems, as different users are likely to have slightly different time delays. However, there are few algorithms, if any, proposed for quasi-synchronous SDMA systems due to the difficulties in time-delay estimation and intersymbol interference (ISI) cancellation.

In this letter, a Rake-based multiuser detection technique which incorporates a new simple time-delay estimation method is proposed for quasi-synchronous SDMA systems operating in multipath channels. The proposed technique is a semiblind technique and consists of three steps. The first step separates the multiuser multipath signal vector into multiuser single-path signal vectors, based on second-order statistics (SOS) of the received signals without knowledge of the channel state information. A simple estimation method exploiting the structural property of the channel matrix is then proposed in the second step to estimate the time delays of all users. The third step combines multiple multiuser single-path signal vectors for signal detection. The advantages of the proposed technique are: 1) perfect synchronization of all users is unnecessary; 2) time

Paper approved by C. Tellambura, the Editor for Modulation and Signal Design of the IEEE Communications Society. Manuscript received January 3, 2006; revised July 3, 2006. This work was supported by the Hong Kong Research Grants Council under Grant 7164/04E.

S. Ma and T.-S. Ng are with the Department of Electrical and Electronic Engineering, The University of Hong Kong, Hong Kong (e-mail: sdma@ eee.hku.hk; tsng@eee.hku.hk).

Y. Zeng is with the Institute for Infocomm Research, A-STAR, Singapore (e-mail: yhzeng@i2r.a-star.edu.sg).

Digital Object Identifier 10.1109/TCOMM.2007.892440 diversity is achieved by the Rake-based receiver for performance enhancement; 3) only the upper bounds of the channel length and the time delays are needed. Simulation results show that the proposed technique performs well and is robust against overestimation of both the maximum channel length and the maximum time delay.

\section{SYSTEM MODEL}

For a $P$-users $M$-receive-antennas quasi-synchronous SDMA system operating in multipath fading channels, the received signal at the $j$ th receive antenna at time $n$ can be expressed as

$y_{j}(n)=\sum_{i=1}^{P} \sum_{l=0}^{L_{i j}} h_{i j}(l) x_{i}\left(n-l-d_{i}\right)+w_{j}(n), j=1,2, \ldots, M$

where $x_{i}(n)$ denotes the transmitted signal of the $i$ th user at time $n$, which is assumed to be independent from each other and satisfy $E\left\{x_{i}(m) x_{j}(n)\right\}=\delta(m-n) \delta(i-j) ; d_{i}$ is the time delay of the $i$ th user signal, $i \in\{1,2, \ldots, P\}$, which is assumed to be not greater than the maximum time delay in this system $d_{P U}$; $w_{j}(n)$ is the additive white Gaussian noise; $h_{i j}(l)$ represents the multipath channel between the transmit antenna of the $i$ th user and the $j$ th receive antenna, which is generally modeled as an $L_{i j}$ th-order finite impulse response (FIR) filter when discretetime representation is employed. Letting $\bar{x}_{i}(n)=x_{i}\left(n-d_{i}\right)$ and $L=\max _{1 \leq i \leq P, 1 \leq j \leq M}\left(L_{i j}\right)$ denote the maximum channel length, the system can be modeled similarly to that in [2] as

$$
\mathbf{y}_{N+1}(n)=\mathbf{H} \overline{\mathbf{x}}_{N+1}(n)+\mathbf{w}_{N+1}(n)
$$

where

$$
\begin{aligned}
\mathbf{y}_{N+1}(n) & =\left[\mathbf{y}(n)^{T}, \mathbf{y}(n-1)^{T}, \cdots, \mathbf{y}(n-N)^{T}\right]^{T} \\
\overline{\mathbf{x}}_{N+1}(n) & =\left[\overline{\mathbf{x}}(n)^{T}, \overline{\mathbf{x}}(n-1)^{T}, \cdots, \overline{\mathbf{x}}(n-L-N)^{T}\right]^{T} \\
\mathbf{w}_{N+1}(n) & =\left[\mathbf{w}(n)^{T}, \mathbf{w}(n-1)^{T}, \cdots, \mathbf{w}(n-N)^{T}\right]^{T}
\end{aligned}
$$

and $\mathbf{H}$ is an $M(N+1) \times P(N+L+1)$ channel convolution matrix, which is similar to $\mathbf{A}$ in [2]. Here we also make a general assumption that the channel convolution matrix $\mathbf{H}$ is of full column rank after removing all-zero columns. In (3), $\mathbf{y}(n)$ is an $M \times 1$ received signal vector, $\overline{\mathbf{x}}(n)$ is a $P \times 1$ transmitted signal vector with time delays, $\mathbf{w}(n)$ is an $M \times 1$ noise vector.

\section{RaKe-Based Multiuser Detection TechniQue}

To simplify the derivation of the algorithm, zero noise is first assumed. The effect of noise on the developed technique is then examined. In the absence of noise, $\mathbf{y}_{N+1}(n)$ can be expressed as

$$
\mathbf{y}_{N+1}(n)=\mathbf{H} \overline{\mathbf{x}}_{N+1}(n) \text {. }
$$




\section{A. Multiuser Single-Path Signal Separation}

The observation vector $\mathbf{y}_{N+1}(n)$ in (4) can also be rewritten as

$$
\mathbf{y}_{N+1}(n)=\mathbf{H} \overline{\mathbf{x}}_{N+1}(n)=\sum_{l=0}^{N+L} \mathbf{H}_{P}(l) \overline{\mathbf{x}}(n-l)
$$

in which $\mathbf{H}_{P}(l)$ denotes a submatrix of $\mathbf{H}$, composed by the columns between the $(l P+1)$ th column and the $(l+1) P$ th column of $\mathbf{H}$. It is obvious that the received signal vector $\mathbf{y}_{N+1}(n)$ is a superposition of $(N+L+1)$ path multiuser signals. Here, as the delay information is embedded in $\bar{x}_{i}(n)$ and the quasi-synchronous system is modeled similarly to that of [2], the ISI cancellation method proposed for synchronous systems in [2] can be applied to separate the single-path signals. From [2], an equalizer $\mathbf{G}_{k}$ is designed as

$$
\mathbf{G}_{k}=\mathbf{U}_{k}-\mathbf{U}_{k+1}, \quad k \geq 0
$$

where $\mathbf{U}_{k}=\mathbf{R}_{Y}(k) \mathbf{R}_{Y}(0)^{\#} \mathbf{R}_{Y}(-k) \mathbf{R}_{Y}(0)^{\#}$, in which $(\cdot)^{\#}$ represents the pseudoinverse and $\mathbf{R}_{Y}(k)$ is defined as $\mathbf{R}_{Y}(k)=$ $E\left\{\mathbf{y}_{N+1}(n+k) \mathbf{y}_{N+1}(n)^{*}\right\}$. Obviously, $\mathbf{R}_{Y}(k)$ is the SOS of the received signal vectors. It has special structures [2] which enable the equalizer $\mathbf{G}_{k}$ to cancel the ISI. When applying the equalizer $\mathbf{G}_{k}$ to the received signal vector $\mathbf{y}_{N+1}(n)$, the $(k+$ 1)th path multiuser signal vector is obtained as [2]

$$
\mathbf{o}_{k}(n)=\mathbf{G}_{k} \mathbf{y}_{N+1}(n)=\mathbf{H}_{P}(k) \overline{\mathbf{x}}(n-k) .
$$

In order to improve the system performance by time diversity, a number of multiuser single-path signal vectors must be separated from $\mathbf{y}_{N+1}(n)$. Multiple equalizers are therefore designed based on (6), with the parameter $k$ having different values. The remaining question is how $k$ should be selected. As the middle columns of $\mathbf{H}$ have large norms, their selection should provide the strongest signal components [2]. Therefore, $k$ is set as $k=k_{o} \pm q, q=0,1,2, \ldots$, where $k_{o}$ equals $\lfloor(N+L+1) / 2\rfloor$ and denotes truncation of the enclosed parameter. Note that $k$ satisfies $0 \leq k \leq N+L-d_{P U}$.

\section{B. Time-Delay Estimation}

The time-delay information is now embedded in each multiuser single-path signal vector $\mathbf{o}_{k}(n), k \in\left\{k_{o}, k_{o} \pm 1, k_{o} \pm\right.$ $2, \cdots\}$ in (7). It can be estimated using any one of them. Apparently, the strongest multiuser single-path signal vector $\mathbf{o}_{k_{o}}(n)=$ $\mathbf{H}_{P}\left(k_{o}\right) \overline{\mathbf{x}}\left(n-k_{o}\right)$ is the best choice for time-delay estimation. To the best of the authors' knowledge, only two pilotbased time-delay estimation methods, the sliding correlation and vector orthogonalization methods, have been proposed [6]. Both of them were derived based on the output signal model, which modeled the delay information in the user signal vector $\overline{\mathbf{x}}\left(n-k_{o}\right)$. However, the sliding correlation method cannot accurately estimate the time delays when the difference between any two delays is less than two symbol periods, and the vector orthogonalization method is rather complicated. As the time-delay information can be modeled either in the user signal vector or in the channel matrix, a simple method to estimate the delay information is proposed here using the structural property of the latter case. It is relatively simple, compared with [6], and it also has the added advantage that it does not require the actual maximum time delay. Only the upper bound is needed.

Rewrite the multiuser single-path signal vector $\mathbf{o}_{k_{o}}(n)$ as

$$
\mathbf{o}_{k_{o}}(n)=\mathbf{H}_{P}\left(k_{o}\right) \overline{\mathbf{x}}\left(n-k_{o}\right)=\overline{\mathbf{H}}_{R E}\left(k_{o}\right) \mathbf{x}_{R E}\left(n-k_{o}\right) .
$$

In (8), $\mathbf{x}_{R E}\left(n-k_{o}\right)=\left[\mathbf{x}\left(n-k_{o}\right)^{T} \mathbf{x}\left(n-k_{o}-1\right)^{T} \cdots \mathbf{x}(n-\right.$ $\left.\left.k_{O}-d_{P U}\right)^{T}\right]^{T}$, where $\mathbf{x}(n)$ is a $P \times 1$ transmitted signal vector without time delays; $\overline{\mathbf{H}}_{R E}\left(k_{o}\right)$ is an $M(N+1) \times\left(d_{P U}+1\right) P$ matrix where the $\left(d_{i} P+i\right)$ th column, $i \in\{1,2, \ldots, P\}$, is the $\left(k_{o} P+i\right)$ th column of $\mathbf{H}$ and other columns are all-zero columns. Namely, the matrix $\overline{\mathbf{H}}_{R E}\left(k_{o}\right)$ has only $P$ nonzero columns on the position $\left(d_{i} P+i\right)$. Time-delay information is now embedded in the channel matrix $\overline{\mathbf{H}}_{R E}\left(k_{o}\right)$. Suppose $\overline{\mathbf{H}}_{R E}\left(k_{o}\right)$ is known; it follows that for each $i$, the norm of the $\left(d_{i} P+i\right)$ th column of $\overline{\mathbf{H}}_{R E}\left(k_{o}\right)$ must be the largest among the $\left(m_{i} P+i\right)$ th columns, $m_{i} \in\left\{0,1, \ldots, d_{P U}\right\}$, because other columns among these $\left(d_{P U}+1\right)$ columns are all-zero columns. With this special structural property, the $\left(m_{i} P+i\right)$ th columns, $m_{i} \in\left\{0,1, \ldots, d_{P U}\right\}$, can be grouped together to form $P$ different groups. Select the column with the largest norm in each group and denote its position in the matrix $\overline{\mathbf{H}}_{R E}\left(k_{o}\right)$ as $K_{i}$, $i \in\{1,2, \ldots, P\}$. The estimated time delay $\hat{d}_{i}$ is therefore obtained by $\left(K_{i}-i\right) / P$.

It is clear that in order to estimate the time delays, knowledge of the matrix $\overline{\mathbf{H}}_{R E}\left(k_{o}\right)$ is required. Obviously, there is no restriction on how $\overline{\mathbf{H}}_{R E}\left(k_{o}\right)$ is to be estimated. However, as the accuracy of the time-delay estimation has a profound effect on the performance of multiuser detection, it is preferred to use some pilots embedded in the signals so that the estimation accuracy can be guaranteed. It is apparent that only $\left(d_{P U}+1\right) P+$ $d_{P U}$ pilots are required to estimate $\overline{\mathbf{H}}_{R E}\left(k_{o}\right)$ based on (8) after multiuser single-path signal separation.

\section{Multipath Combining}

In the proposed Rake-based receiver, $2 m+1$ fingers are included, each corresponding to a different delay of $\mathbf{y}_{N+1}(n)$ equalized by a different equalizer. The $q$ th finger output is given by $\mathbf{o}_{k_{o}-q}(n-q)=\mathbf{G}_{k_{o}-q} \mathbf{y}_{N+1}(n-q)=\mathbf{H}_{P}\left(k_{o}-q\right) \overline{\mathbf{x}}(n-$ $\left.k_{o}\right), q=0, \pm 1, \pm 2, \ldots, \pm m$. It is obvious that $\mathbf{o}_{k_{o}-q}(n-q)$ is the $\left(k_{o}-q+1\right)$ th path multiuser signal. The $2 m+1$ path multiuser signals are then combined by the combining weights $\mathbf{V}_{k_{o}-q}$. The output of the Rake-based receiver, $\mathbf{z}(n)$, is therefore given by

$$
\mathbf{z}(n)=\sum_{q=-m}^{m} \mathbf{V}_{k_{o}-q} \mathbf{o}_{k_{o}-q}(n-q)=\overline{\mathbf{V}}_{m} \overline{\mathbf{H}}_{P, m} \overline{\mathbf{x}}\left(n-k_{o}\right)
$$

where

$$
\begin{aligned}
& \overline{\mathbf{H}}_{P, m}= {\left[\begin{array}{llll}
\mathbf{H}_{P}\left(k_{o}+m\right)^{T} & \ldots & \mathbf{H}_{P}\left(k_{o}\right)^{T} & \ldots \\
\mathbf{H}_{P}\left(k_{o}-m\right)^{T}
\end{array}\right]^{T} } \\
& \overline{\mathbf{V}}_{m}=\left[\begin{array}{lllll}
\mathbf{V}_{k_{o}+m} & \ldots & \mathbf{V}_{k_{o}} & \ldots & \mathbf{V}_{k_{o}-m}
\end{array}\right] .
\end{aligned}
$$

By choosing the combining weight vector based on least-squares criteria as $\overline{\mathbf{V}}_{m}=\left(\overline{\mathbf{H}}_{P, m}^{*} \overline{\mathbf{H}}_{P, m}\right)^{-1} \overline{\mathbf{H}}_{P, m}^{*}$, the output signal becomes $\mathbf{z}(n)=\overline{\mathbf{x}}\left(n-k_{o}\right)$. Apparently, it is the estimated multiuser signal vector with time-delay information 


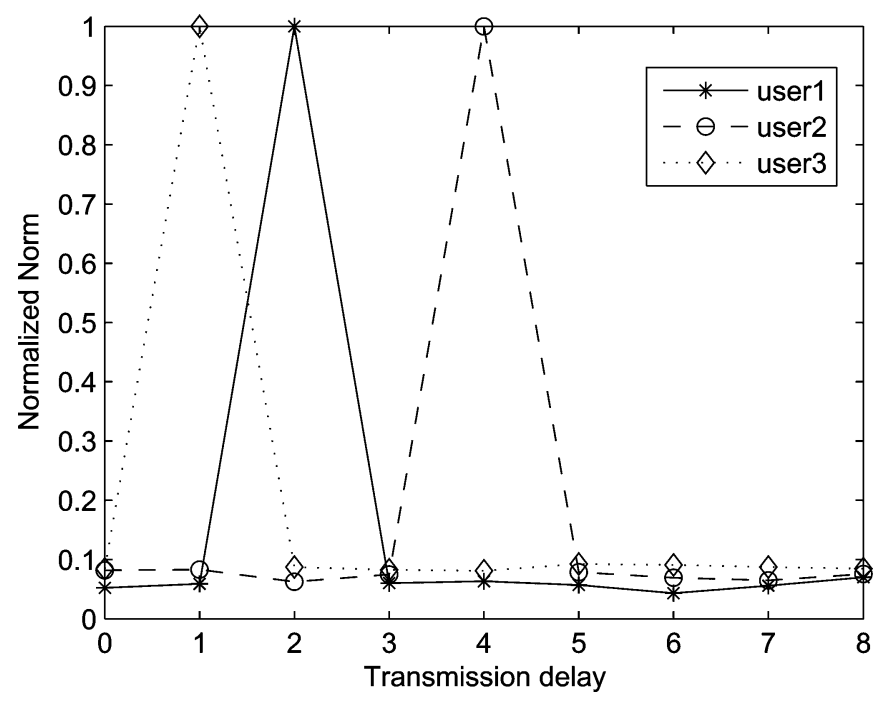

Fig. 1. Time-delay estimation $\left(d_{1}=2, d_{2}=4\right.$, and $\left.d_{3}=1 ; \mathrm{SNR}=20 \mathrm{~dB}\right)$.

embedded. It follows that with the time delays estimated in Step 2, the transmitted multiuser signals are obtained.

In order to compute the combining weight vector $\overline{\mathbf{V}}_{m}$, the channel gain matrices $\mathbf{H}_{P}\left(k_{o}+q\right), q \in\{0, \pm 1, \pm 2, \ldots, \pm m\}$ are required. We recall that pilots have been inserted into each user's signal to estimate the time delays. They can also be used in this step to estimate $\mathbf{H}_{P}\left(k_{o}+q\right)$ from (7), based on leastsquares criteria.

\section{Channel Noise Consideration}

In the presence of noise, $\mathbf{y}_{N+1}(n)$ is given by (2). Let the variance of noise be $\sigma^{2}$. The autocorrelation matrix of $\mathbf{y}_{N+1}(n)$ is

$$
\begin{aligned}
\mathbf{R}_{Y}(k) & =E\left\{\mathbf{y}_{N+1}(n+k) \mathbf{y}_{N+1}(n)^{*}\right\} \\
& =\mathbf{H J}^{k P} \mathbf{H}^{*}+\sigma^{2} \mathbf{J}^{k M}, \quad k=0, \pm 1, \pm 2, \ldots
\end{aligned}
$$

Generally, $\sigma^{2}$ is unknown and can be estimated from the singular value decomposition of $\mathbf{R}_{Y}(0)$, where $\mathbf{R}_{Y}(0)=\mathbf{H} \mathbf{H}^{*}+$ $\sigma^{2} \mathbf{I}$. The noise contribution can then be subtracted from $\mathbf{R}_{Y}(k)$, and therefore, it has no impact on the equalizers $\mathbf{G}_{k}$. In this case, the multiuser single-path signal vectors $\mathbf{o}_{k}(n)$ become $\mathbf{o}_{k}(n)=$ $\mathbf{G}_{k} \mathbf{y}_{N+1}(n)=\mathbf{H}_{P}(k) \overline{\mathbf{x}}(n-k)+\mathbf{G}_{k} \mathbf{w}_{N+1}(n)$. Taking into account the noise contribution $\mathbf{G}_{k} \mathbf{w}_{N+1}(n)$ in $\mathbf{o}_{k}(n)$, the combining weight matrix $\overline{\mathbf{V}}_{m}$ can be designed based on the minimum mean-square error (MMSE) criterion [7].

\section{EXAMPLES AND SimULATION RESUltS}

First, a quasi-synchronous SDMA system with $P=3$ users and $M=3$ receive antennas operating in single-path channels was used as an example to illustrate the proposed time-delay estimation method. The synchronization window $d_{P U}$ was set to eight, and the time delays were arbitrarily selected for illustration as follows: $d_{1}=2, d_{2}=4$, and $d_{3}=1$. For single-path channels, the time-delay estimation method in Step

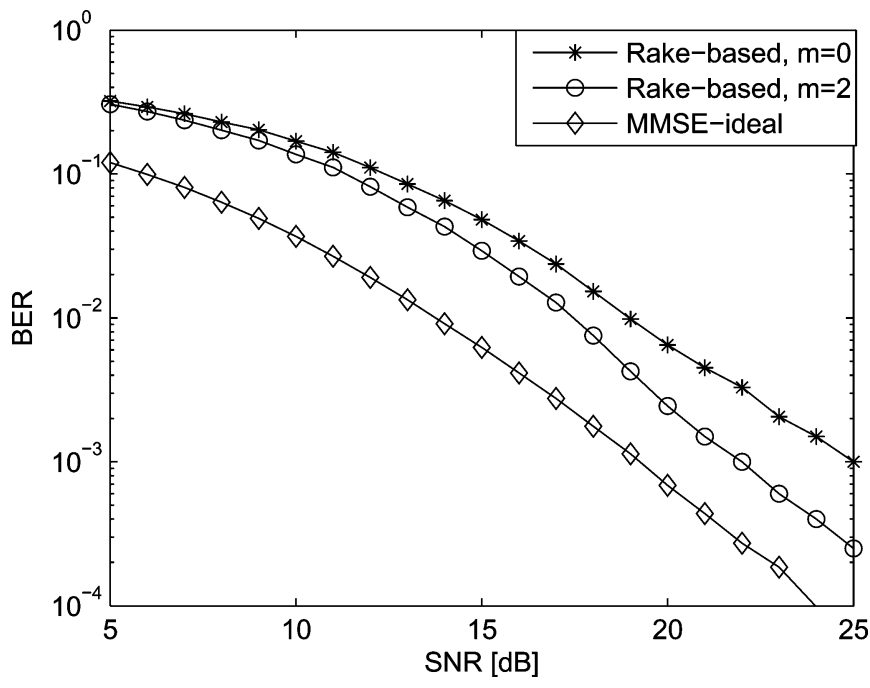

Fig. 2. BER versus SNR $\left(d_{P U}=2\right.$ and $\left.L=3\right)$.

2 can be directly applied by remodeling the channel matrix $\mathbf{h}(0)$ as $\mathbf{h}_{R E}(0)$. Results are shown in Fig. 1. The solid line, the dashed line, and the dot line, respectively, indicate the normalized norms of the $\left(m_{i} P+1\right)$ th columns, the $\left(m_{i} P+2\right)$ th columns, and the $\left(m_{i} P+3\right)$ th columns, $m_{i} \in\left\{0,1, \ldots, d_{P U}\right\}$, in the estimated matrix $\hat{\mathbf{h}}_{R E}(0)$. Each group's norms are normalized by the maximum norm in the group. It is apparent that the peaks are very sharp and the time delays can be accurately estimated by the positions of the peaks, even if the condition that the difference between any two delays is not less than two symbol periods is unsatisfied. Note that this condition is required for the sliding correlation method in [6].

Second, the performance of the proposed Rake-based technique was investigated using a three-users five-receive-antennas quasi-synchronous SDMA system with arbitrarily selected time delays $\left(d_{1}=0, d_{2}=1\right.$, and $\left.d_{3}=2\right)$ as an example. The maximum time delay $d_{P U}$ was set to two and the maximum channel length $L$ was equal to three. The autocorrelation of the received signal vector was computed as $\mathbf{R}_{Y}(k) \approx 1 / N_{s} \sum_{n=1}^{N_{s}} \mathbf{y}_{N+1}(n+k) \mathbf{y}_{N+1}(n)^{*}$ where $N_{s}$ was chosen as 500 in our simulations. The performance measure, BER, was computed by averaging the results among all users over 1000 simulation runs, each with 500 symbols. Two Rake-based receivers were designed with the parameter $m=0$ (corresponding to single-path receiver) and $m=2$ (arbitrarily selected for illustration). For comparison purposes, the MMSE multiuser detector with perfect time delay and channel state information (MMSE-ideal) was also implemented. Fig. 2 shows the performance of various algorithms under consideration when the maximum time delay $d_{P U}$ and the maximum channel length $L$ are known exactly. It is obvious that when the parameter $m$ increases from zero to two, the performance of the proposed Rake-based algorithm is improved by multipath combining. This result demonstrates the effect of time diversity. Note that the performance of the MMSE-ideal algorithm is the best that linear multiuser detection algorithms 


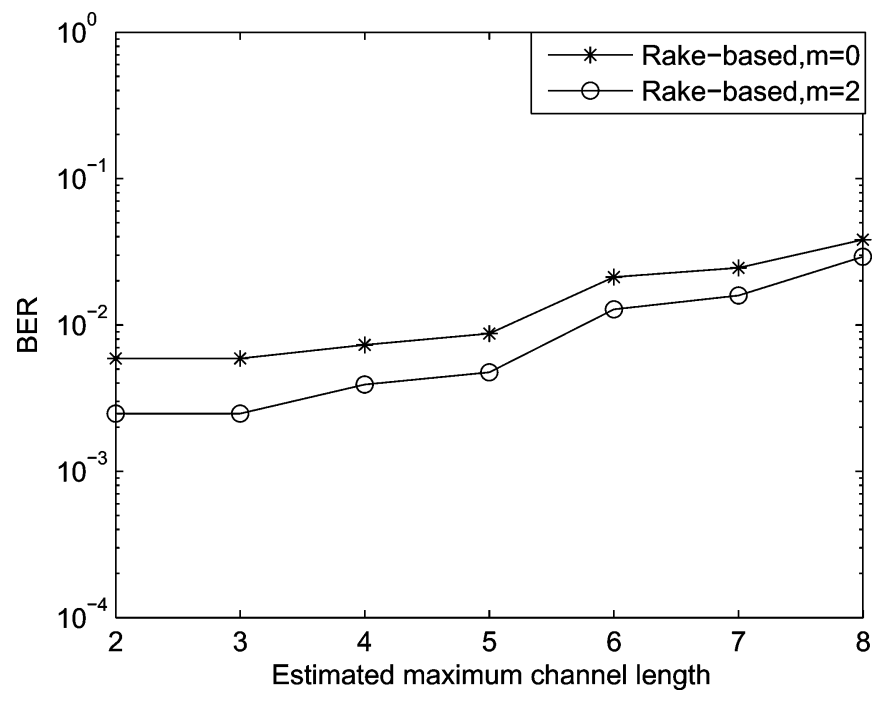

Fig. 3. BER versus the estimated maximum channel length $\hat{L}$ (SNR $=20 \mathrm{~dB}$; $d_{P U}=2 ; L=3$ ).

can achieve because the channel convolution matrix and the time delays are assumed perfectly known. Next, the estimated maximum channel length $\hat{L}$ was varied from two to eight (SNR $=20 \mathrm{~dB}$ ) to test its effect on the proposed algorithm. Results are shown in Fig. 3. The BER of the proposed algorithm only increases marginally when the estimated maximum channel length increases. Finally, Fig. 4 shows the performance of the Rake-based algorithm for the same system when the maximum time delay $d_{P U}$ is overestimated. The results also indicate that the proposed algorithm is robust to the maximum time delay overestimation.

\section{CONCLUSION}

A three-step Rake-based multiuser detection technique for quasi-synchronous SDMA systems has been proposed. Multipath signals have been exploited to achieve time diversity, therefore improving the performance. A simple time-delay estimation method, which exploits the structural property of the channel matrix, has been proposed. It has been shown that knowledge of the channel length and the time delays are not required, which renders the technique more practical. Simulation

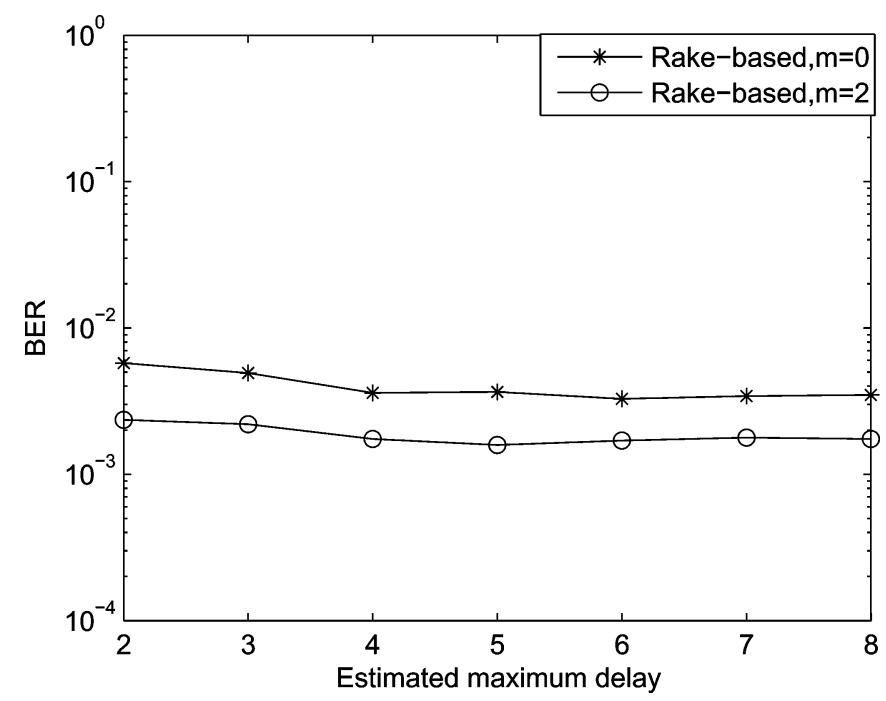

Fig. 4. BER versus the estimated maximum delay $\hat{d}_{P U}\left(\mathrm{SNR}=20 \mathrm{~dB} ; d_{P U}=\right.$ $2 ; L=3)$.

results have demonstrated that the proposed technique achieves good performance and is robust against overestimation of the maximum channel length and the maximum time delay.

\section{REFERENCES}

[1] J. H. Winters, J. Salz, and R. D. Gitlin, "The impact of antenna diversity on the capacity of wireless communication systems," IEEE Trans. Commun., vol. 42, no. 2-4, pp. 1740-1751, Feb.-Apr. 1994.

[2] J. Zhu, Z. Ding, and X.-R. Cao, "Column-anchored zeroforcing blind equalization for multiuser wireless FIR channels," IEEE J. Sel. Areas Commun., vol. 17, no. 2, pp. 411-423, Mar. 1999.

[3] J. K. Tugnait, "Blind estimation and equalization of MIMO channels via multidelay whitening," IEEE J. Sel. Areas Commun., vol. 19, no. 8, pp. 1507-1519, Aug. 2001

[4] C. L. Miller, D. P. Taylor, and P. T. Gough, "Estimation of co-channel signals with linear complexity," IEEE Trans. Commun., vol. 49, no. 11, pp. 1997-2005, Nov. 2001

[5] Y. Hua, S. An, and Y. Xiang, "Blind identification of FIR MIMO channels by decorrelating subchannels," IEEE Trans. Signal Process., vol. 51, no. 5, pp. 1143-1155, May 2003.

[6] T. Nishimura, Y. Tanabe, T. Ohgane, Y. Ogawa, Y. Doi, and J. Kitakado, "Spatial domain interference canceler using multistage adaptive array with precise timing estimation," in Proc. IEEE Veh. Technol. Conf., Sep. 2000, vol. 2, pp. 719-724.

[7] S. M. Kay, Fundamentals of Statistical Signal Processing: Estimation Theory. Englewood Cliffs, NJ: Prentice-Hall, 1993. 